\author{
Won-Bae Chang, Ho-Seong Han*, Jai \\ Young Cho, Yoo-Seok Yoon, Young \\ Rok Choi and Bo-Ram Lee \\ Department of Surgery Seoul National University \\ College of Medicine, Seoul National University \\ Bundang Hospital, Seongnam, South Korea \\ Dates: Received: 08 April, 2016; Accepted: 28 April, \\ 2016; Published: 30 April, 2016 \\ *Corresponding author: Ho-Seong Han, \\ Department of Surgery, Seoul National University \\ College of Medicine, Seoul National University, \\ Bundang Hospital, Seongnam, South Korea, 173, 82 \\ Gumi-ro, Bundang-gu, Seongnam, Gyeonggi, South \\ Korea, 463-707, Tel: 82-31-787-7091 \\ E-mail: hanhs@snubh.org \\ www.peertechz.com \\ ISSN: 2455-2283
}

\author{
Case Report
}

\section{ABO-incompatible living donor liver transplantation in a patient with a history of platelet transfusion- related anaphylaxis}

\section{Abbreviations}

ATR: Allergic Transfusion Reaction; CT: Computed Tomography; FFP: Fresh Frozen Plasma; HCC: Hepatocellular Carcinoma; Ig: Immunoglobulin; IVIG: Intravenous Gamma Globulin; LDLT: Living Donor Liver Transplantation; RFA: Radiofrequency Ablation; TACE: Transarterial Chemoembolization;

\section{Introduction}

Allergic transfusion reactions (ATR) are common complications after blood transfusion, and occur in up to $10.2 \%$ of patients who receive blood products via transfusion $[1,2]$. The clinical symptoms range from fever, hives, and edema to dyspnea, tachycardia, and hypotension. However, anaphylaxis and severe ATRs are rare complications. Anaphylaxis is more common after the transfusion of plasma-containing products, with an incidence of 1 per 10,000-33,000 units of platelets or 1 per 29,000-50,000 units of fresh frozen plasma (FFP) as compared with 1 in 50,000-200,000 units of red blood cells transfused [1-3]. Although transfusion-related anaphylaxis is rare, it is associated with severe clinical outcomes, including death [4].

Liver transplantation is one of the most effective treatments for patients with end-stage liver diseases, such as hepatitis B- or C-induced liver cirrhosis or hepatocellular carcinoma (HCC). However, owing to the scarcity of cadaveric donors relative to the number of patients waiting for liver transplantation, living donors have gained traction as an alternative. In recent decades, surgeons have focused on $\mathrm{ABO}$-incompatible liver transplantations, and the outcomes of this procedure have improved significantly with accumulating knowledge of balanced immunosuppression protocols [5].

Many candidates for liver transplantation have portal vein hypertension, splenomegaly, and thrombocytopenia. They also have a prolonged prothrombin time caused by deficient production of coagulation factors. Accordingly, these patients have much a higher risk of bleeding during their illness and blood transfusion is frequently required in their resuscitation. Furthermore, liver transplantation is a complicated procedure that is expected to cause significant blood loss [6], even when the surgeon uses many approaches and techniques to minimize this loss. Therefore, transfusion of multiple units of blood products is necessary during liver transplantation.

Here, we report a patient with hepatitis B virus-induced liver cirrhosis and HCC who experienced platelet transfusion-related anaphylaxis after successful ABO-incompatible living donor liver transplantation (LDLT).

\section{Case Presentation}

A 64-year-old male with hepatitis B-induced liver cirrhosis and HCC presented at an emergency room in April, 2013 with abdominal pain and melena. He was previously diagnosed with esophageal varix Grade II [7], caused by liver cirrhosis and a duodenal bulb ulcer. His white blood cell count, hemoglobin concentration, and platelet count were $3.39 \times 10^{3} / \mu \mathrm{L}, 14.6 \mathrm{~g} / \mathrm{dL}$, and $33 \times 10^{3} / \mu \mathrm{L}$, respectively.

He was transfused with 6 units of platelets, which resulted in an acute hypotensive response (blood pressure 77/53 $\mathrm{mmHg}$ ) and hypoxia (oxygen saturation $80 \%$ ). He was treated with intravenous epinephrine $(1 \mathrm{mg})$, chlorpheniramine $(4 \mathrm{mg})$, fluid resuscitation, and supplemental oxygen. Department of allergy medicine was contacted and the patient's serum immunoglobulin concentrations were checked as follows: IgG, $1350 \mathrm{mg} / \mathrm{dL}$ (normal range 700-1700 $\mathrm{mg} / \mathrm{dL}$ ); IgA, $179 \mathrm{mg} / \mathrm{dL}$ (normal range 90-400 mg/dL); IgM, 122 $\mathrm{mg} / \mathrm{dL}$ (normal range $45-230 \mathrm{mg} / \mathrm{dL}$ ); IgE, $144.7 \mathrm{mg} / \mathrm{dL}$ (normal range $0-100 \mathrm{mg} / \mathrm{dL}$ ). Owing to suspected platelet transfusionrelated anaphylaxis, it was recommended that the patient should undergo slower transfusion and apheresis platelets need to be used if 
transfusion was required again. His vital signs stabilized within $6 \mathrm{~h}$ of starting resuscitation. The next day, his condition had improved and he was discharged from the Emergency Room.

In September 2014, the patient was admitted for radiofrequency ablation (RFA) and trans-arterial chemoembolization (TACE) to treat newly developed HCC lesions in segments 6 and 8 of the liver. His serum platelet level was $23 \times 10^{3} / \mu \mathrm{L}$ at admission. Because of the high risk of bleeding during RFA and TACE, he underwent apheresis platelet transfusion. One hour after transfusion, he became febrile $\left(38.5^{\circ} \mathrm{C}\right)$ and started shivering. RFA and TACE were abandoned and he was managed with antipyretics. His symptoms subsided the next day. The event was considered to be a nonhemolytic febrile reaction. Five days later, TACE and percutaneous ethanol injection were performed instead of RFA.

We continued to monitor the patient's HCC and he was readmitted in April 2015 for treatment of HCC recurrence and deteriorating liver function. We discussed with the patient and his family that liver transplantation was an option. His 19-year-old son was determined to be a living donor with an $\mathrm{ABO}$-incompatible blood type (recipient: $\mathrm{Rh}+\mathrm{O}$; donor: $\mathrm{Rh}+\mathrm{A}$ ). The recipient underwent preconditioning therapy for ABO-incompatible LDLT according to the protocol used at Seoul National University Bundang Hospital, Seongnam, South Korea (Figure 1).

The patient's serum isoagglutinin titers were measured (anti-A IgG: 128; anti-A IgM: 64) and plasma exchange was performed twice, until the anti-A Ig titers were reduced to $1: 16$ or lower (anti-A IgG: 16; Anti-A IgM: 8) before transplantation. Washed apheresis platelet transfusion preceded each plasma exchange. The patient did not exhibit any allergic event after the first platelet transfusion, but he developed shivering and became febrile $\left(39.5^{\circ} \mathrm{C}\right) 1 \mathrm{~h}$ after the second platelet transfusion. Therefore, he was managed with antipyretics and his symptoms subsided within a few hours. The second plasma exchange was performed without further allergic events. Because his prothrombin time (international normalized ratio) increased from 2.03 before plasma exchange to 4.62 after plasma exchange, 1 unit of

\section{SNUBH Protocol for ABO incompatible LT}

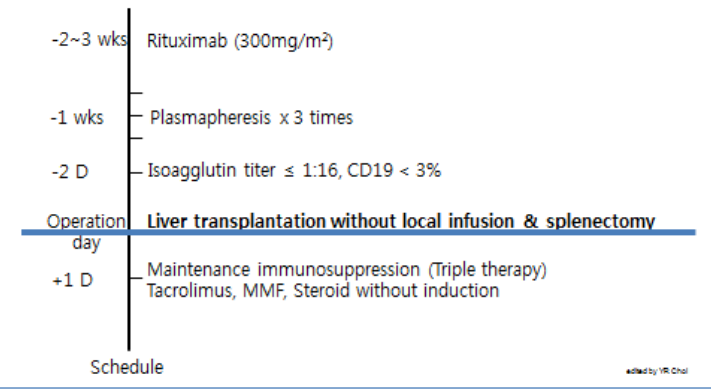

$*$ Patient with high titer even after multiple session of plasmapheresis $\rightarrow$ intraoperative IMG $0.8 \mathrm{~g} / \mathrm{Kg}$

Figure 1: Protocol for ABO-incompatible living donor liver transplantation. d, day; LT, liver transplantation; MMF, mycophenolate mofetil; SNUBH, Seoul National University Bundang Hospital; wk, week; IVIG, intravenous gamma globulin.
FFP was given and was tolerated. A single dose of rituximab (300 mg/ $\mathrm{m}^{2}$ ) was administered 2 weeks before transplantation.

Preoperative computed tomography (CT) revealed a splenic artery aneurysm that had increased in size from $3 \mathrm{~cm}$ to $3.6 \mathrm{~cm}$, compared with the previous CT. The aneurysm and splenic artery were embolized to prevent bleeding from this site. However, during surgery, it was noted that the splenic artery was incompletely embolized, so splenectomy was performed. Perioperative intravenous $\gamma$-globulin $(0.8 \mathrm{~g} / \mathrm{kg}$; IVIG) was administered to the patient in the hepatic phase and was continued until 2 days after transplantation.

The patient was transfused with 23 units of red blood cells, 27 units of FFP, and 3 units of apheresis platelets during surgery and there were no transfusion-related allergic reactions. LDLT was completed without complications.

\section{Discussion}

ATRs sometimes occur after platelet transfusion. The risk of an allergic reaction after platelet transfusion ranged from $0.09 \%$ to $21 \%$ in prior studies $[8,9]$. Its manifestations are broad, and range from mild skin reactions such as pruritus and urticaria to systemic reactions such as bronchoconstriction, hypotension, and shock.

The pathogenesis of ATRs is very heterogeneous. It is most likely caused by (i) IgE and IgG antibodies in the recipient targeting plasma proteins in the transfused blood component, and (ii) cytokines, chemokines, and histamine in the platelet products. The most common type of anaphylactic reaction is induced by IgA deficiency in the recipient and the subsequent formation of antiIgA [10]. However, Matsuyama et al. found no antibodies against plasma proteins, including IgA, in the serum of most patients with severe ATRs after platelet transfusions [11]. Based on the clinical and pathologic findings, we think that the most likely etiology of our patient's transfusion reaction was an anaphylactic reaction, even though his serum Ig concentrations were in normal ranges except for a mild elevation of IgE.

Because the patient had experienced an anaphylactic episode after platelet transfusion, he was to be given washed apheresis platelets to prevent subsequent ATRs, whenever transfusion was required, because this was reported to be effective in previous studies $[12,13]$.

The patient also received IVIG perioperatively in accordance with the protocol for ABO-incompatible LDLT. IVIG has potent immunomodulatory effects and is beneficial in the treatment of various autoimmune and inflammatory disorders [14]. The proposed mechanisms of action of IVIG on humoral reactions include promotion of B cell or plasma cell apoptosis through the Fc-receptor dependent pathway, and inhibition of alloreactive T cell-mediated or complement-mediated allograft injury, although these possibilities have not yet been confirmed [15]. Additionally, IVIG might prevent further anaphylactic reactions after postoperative platelet transfusions, even though the mechanism by which it prevents these anaphylactic reactions is unknown $[16,17]$.

Splenectomy is an integral part of the protocol for ABOincompatible LDLT at many institutions because the spleen is the site of $\mathrm{B}$ cell maturation and antibody production $[18,19]$. 
However, splenectomy is associated with many procedure-related complications including portal vein thrombosis, pancreatic fistula, and sepsis [20,21]. Rituximab is a monoclonal chimeric humanmurine anti-CD20 antibody that destroys $\mathrm{B}$ cells by promoting complement-dependent cellular cytotoxicity. It removes CD20positive $\mathrm{B}$ cells from the circulation and the lymphoid tissues of the spleen. Therefore, rituximab acts as a form of chemical splenectomy [22]. Studies have shown that preoperative administration of rituximab may replace splenectomy [19,23,24]. Considering this benefit of rituximab in terms of spleen preservation, embolization of a splenic artery aneurysm was performed to preserve the spleen before transplantation. During surgery, however, it was confirmed that the splenic artery was incompletely embolized, permitting blood flow into the splenic artery. This raised some concern of a risk of bleeding from the incompletely embolized splenic artery aneurysm. After evaluating the risks and benefits, splenectomy was performed. Follow-up laboratory tests showed that because his serum platelet count had increased to a sufficient level, platelet transfusion was not required in the immediate postoperative period.

\section{Conclusion}

It is challenging to perform LDLT, which carries a high risk of bleeding, in patients with a history of platelet transfusion-related anaphylaxis. In this patient, the use of IVIG, steroids, apheresis platelet transfusion, rituximab, and splenectomy avoided anaphylaxis during ABO-incompatible LDLT.

\section{Authorship Contribution}

Dr. Chang wrote and edited the case report. Dr. Han reviewed and edited the case report. Dr. Cho, Dr. Yoon, and Dr. Choi were involved in the surgical procedures, the patient's care plan, and reviewed the case reported. Dr. Lee contributed to data collection.

\section{Consent}

The patient gave consent for publishing this manuscript. All efforts were given to keep maintain the patient's anonymity.

\section{References}

1. Stainsby D1, Jones H, Asher D, Atterbury C, Boncinelli A, et al. (2006) Serous hazards of transfusion: a decade of hemovigilance in the UK. Transfusion Medicine Reviews 20: 273-282.

2. Paroskie A, Booth GS (2014) Transfusion related anaphylaxis during orthotopic liver transplantation. Transfusion Apheresis Science 50: 253-254.

3. Domen RE, Hoeltge GE (2003) Allergic transfusion reactions: an evaluation of 273 consecutive reactions. Arch Pathology Lab Medicine127: 316-320.

4. (2012) Administration US FaD. Fatalities reported to FDA following blood collection and transfusion: annual summary for fiscal year 2012

5. Tanabe M, Kawachi S, Obara H, Shinoda M, Hibi T, et al. (2010) Current progress in ABO-incompatible liver transplantation. Eur J clin Invest 40: 943949.

6. De Boer MT, Molenaar IQ, Hendriks HG, Slooff MJ, Porte RJ (2005)
Minimizing blood loss in liver transplantation: Progress through research and evolution of techniques. Digestive Surgery 22: 265-275.

7. Kane L, Kahaleh M, Shami VM, Caldwell SH, Berg CL, et al. (2005) Comparison of the grading of esophageal varices by transnasal endoluminal ultrasound and esophagogastroduodenoscopy. Clin Gastroenterol Hepatol 3: 806-810.

8. Terrence LG, Scott $\mathrm{CH}$ (2007) Acetaminophen and diphenhydramine premedication for allergic and febrile nonhemolytic transfusion reactions: good prophylaxis or bad practice? Transfus Med Rev 21: 1-12.

9. Volker Kiefel (2008) Reaction Induced by Platelet Transfusions. Transfus Med Hemother 35: 354-358.

10. Sandler SG, Mallory D, Malamut D, Eckrich R (1995) IgA anaphylactic transfusion reactions. Transfus Med Rev 9: 1-8.

11. Matsuyama N, Yasui K, Amakishi E, Hayashi T, Kuroishi A, et al. (2015) The IgE-dependent pathway in allergic transfusion reactions: involvement of donor blood allergens other than plasma proteins. Int J Hematol 102: 93-100.

12. Tobian AA, Savage WJ, Tisch DJ, Thoman S, King KE, et al. (2011) Prevention of allergic transfusion reactions to platelets and red blood cells through plasma reduction. Transfusion 51: 1676-1683.

13. Kim H, Choi J, Park KU, Kim HS, Min YH, et al. (2012) Anaphylactic Transfusion Reaction in a Patient with Anhaptoglobinemia: The First Case in Korea. Ann Lab Med 32: 304-306.

14. Jordan S, Cunningham-Rundles C, McEwan R (2003) Utility of intravenous immune globulin in kidney transplantation: efficacy, safety, and cost implications. Am J Transplant 3: 653-664.

15. Ikegami T, Yoshizumi T, Soejima Y, Uchiyama H, Shirabe K, et al. (2016) Feasible usage of $A B O$ incompatible grafts in living donor liver transplantation. Hepatobiliary Surg Nutr 5: 91-97.

16. Richard T, Barton K, Christopher AT (2012) Diagnosis, Treatment, and Reporting of Adverse Effects of Transfusion. Lab Medicine 2012: 217-231.

17. Hirayama $F$ (2013) Current understanding of allergic transfusion reactions: incidence, pathogenesis, lab tests, prevention and treatment. $\mathrm{Br} \mathrm{J}$ Haematol 160: 434-444.

18. Egawa H, Teramukai S, Haga H, Tanabe M, Fukushima M, et al. (2008) Present status of $\mathrm{ABO}$-incompatible living donor liver transplantation in Japan. Hepatology 47: 143-152.

19. Raut V, Mori A, Kaido T, Ogura Y, Taku I, et al. (2012) Splenectomy does not offer immunological benefits in $\mathrm{ABO}$-incompatible liver transplantation with a preoperative rituximab. Transplantation 93: 99-105.

20. Pan C, Shi Y, Zhang JJ, Deng YL, Zheng H, et al. (2009) Single-center experience of 253 portal vein thrombosis patents undergoing liver transplantation in China. Transplant Proc 41: 3761-3765.

21. Troisi R, Hesse UJ, Decruyenaere J, Morelli MC, Palazzo U, et al. (1999) Functional, liver-threatening disorders and splenectomy following liver transplantation. Clin Transplant 13: 380-388.

22. Pescovitz MD (2006) Rituximab, an anti-CD20 monoclonal antibody: history and mechanism of action. Am J Transplant 6: 859-866.

23. Egawa H, Teramukai S, Haga H, Tanabe M, Mori A, et al. (2014) Impact of rituximab desensitization on blood-type-incompatible adult living donor liver transplantation: a Japanese multicenter study. Am J Transplant 14: 102-114.

24. Lee J, Lee JG, Lee JJ, Kim MS, Ju MK, et al. (2008) Results of ABOIncompatible Liver Transplantation Using a Simplified Protocol at a Single Institution. Transplant Proc 47: 723-726.

Copyright: (c) 2016 Chang WB, et al. This is an open-access article distributed under the terms of the Creative Commons Attribution License, which permits unrestricted use, distribution, and reproduction in any medium, provided the original author and source are credited. 